\title{
Rotational stability and visual performance 3 months after bilateral implantation of a new toric extended range of vision intraocular lens
}

This article was published in the following Dove Press journal:

Clinical Ophthalmology

\author{
Kjell Gunnar Gundersen \\ Ifocus Øyeklinikk, Haugesund, Norway
}

Correspondence: Kjell Gunnar

Gundersen

Ifocus Øyeklinikk AS, Sørhauggata I I I,

5527 Haugesund, Norway

Tel +4752808900

Email kg@ifocus.no
Purpose: To evaluate the clinical outcomes after implantation of Symfony ${ }^{\circledR}$ toric extended range of vision (ERV) intraocular lenses (IOLs) in subjects with preoperative corneal astigmatism.

Setting: Ifocus Øyeklinikk, Haugesund, Norway.

Design: Prospective non-comparative study.

Patients and methods: The study population consisted of 30 patients, with preoperative corneal astigmatism between 0.88 and $3.29 \mathrm{D}$, undergoing bilateral cataract removal or refractive lens exchange with implantation of Tecnis Symfony toric ERV IOLs. Main outcome measures were visual acuity, refraction, IOL axis rotational stability, patient and surgeon satisfaction, photic phenomena, and quality of life at 3 months.

Results: Postoperatively, mean binocular uncorrected distance, intermediate, and near visual acuities $(\log$ MAR) were $-0.05 \pm 0.11,-0.03 \pm 0.08$, and $0.09 \pm 0.10$, respectively. A significant reduction in manifest cylinder $(P<0.001)$ and spherical equivalent $(P=0.001)$ was found after 3 months. Monocular and binocular corrected distance visual acuity also improved significantly with surgery $(P<0.001)$. In terms of binocular uncorrected intermediate and near visual acuity, a total of $96 \%$ and $82 \%$ of patients achieved $0.1 \log$ MAR (Snellen: $20 / 25$ ) or better, respectively. Toric IOL axis showed a median rotation of 2.0 degrees (range $0.0-16.0$ degrees) from the end of surgery to 3 months. The median patient satisfaction scores for distance, intermediate, and near vision were 9.0,9.0, and 8.0, respectively. Fifty percent of patients reported complete spectacle independence and most patients (89\%) were not bothered by any photic phenomena.

Conclusion: Bilateral implantation of the Symfony toric ERV IOL appeared to be safe and achieved good visual performance and high satisfaction.

Keywords: rotational stability, toric intraocular lens, extended range of vision

\section{Introduction}

Multifocal intraocular lenses (IOLs) represent a safe and effective solution to achieve spectacle independence after cataract surgery or refractive lens exchange, providing a surgical option for presbyopia correction with high patient satisfaction. ${ }^{1}$ Nowadays, there are various optical designs available for multifocal IOLs, like refractive, diffractive, and hybrid refractive-diffractive lenses. All of them use the principle of simultaneous vision, in which light is split into two or more focal points, requiring the patient to concentrate on multiple coexisting retinal images to undertake a visual task at a specific distance. The split of light has been shown to reduce contrast sensitivity and increase the risk of disturbing photic phenomena. ${ }^{1-3}$ Additionally, traditional multifocal IOLs showed weaknesses in providing satisfactory intermediate vision., To minimize these drawbacks and to enhance the intermediate focal plane, modern 
enhanced depth of focus (EDOF) and extended range of vison (ERV) lenses were developed, incorporating new technical features in their optical designs.

The Tecnis Symfony ${ }^{\circledR}$ IOL (Johnson \& Johnson Vision, Santa Ana, CA, USA) was the first ERV IOL approved by the US Food and Drug Administration in July 2016. To enhance contrast sensitivity, the IOL optic technology relies on an anterior wavefront-designed aspheric surface to compensate the average positive spherical aberration of the cornea. ${ }^{6}$ Additionally, the posterior surface incorporates a proprietary diffractive design, called echelette, which improves contrast sensitivity using achromatic technology for the correction of chromatic aberration. ${ }^{7,8}$ Unlike traditional multifocal lenses, the echelette optic design of Symfony IOLs provides a single elongated focal zone as opposed to multiple distinct foci, with the aim to generate less dysphotopsia. ${ }^{9}$

Theoretical research found that the correction of both spherical and chromatic aberration of the ocular system lead to superior retinal image quality and therefore can improve visual performance. ${ }^{10}$

At the same time, the results of several clinical studies indicate that implantation of Tecnis Symfony IOLs offer an effective way for visual rehabilitation at far and intermediate distances as well as functional near vision. ${ }^{11-18}$ Patients reported a high rate of satisfaction and spectacle independence ${ }^{13,14,16,18}$ also in the case of previous multifocal ablation excimer laser surgery. ${ }^{19}$

Especially with diffractive lenses, postoperative residual refractive astigmatism significantly affects the visual outcomes. ${ }^{20}$ According to Hoffmann and Hütz, ${ }^{21}$ the prevalence of corneal astigmatism greater or equal to 1.00 diopters (D) in presbyopic patients is $36 \%$. The introduction of the toric version of the Tecnis Symfony ERV IOL now offers the opportunity to simultaneously correct patients' preexisting corneal astigmatism.

The aim of this study was to investigate the rotational stability, visual performance, refractive predictability, patient and surgeon satisfaction as well as spectacle independence 3 months after implantation of the new Tecnis Symfony toric ERV IOL.

\section{Patients and methods Patient population}

This prospective non-comparative monocentric study was performed at the Ifocus Eye Clinic, Haugesund, Norway. All patients underwent bilateral non-sequential phacoemulsification between September 2015 and April 2017 with implantation of toric ERV IOLs (Tecnis Symfony toric; Johnson \& Johnson Vision).

Included were patients with age-related cataract or presbyopic patients suitable for refractive lens exchange (RLE) with a demand for postoperative spectacle independence. They had to have regular corneal astigmatism between 0.75 diopters (D) and $3.5 \mathrm{D}$, and a visual potential for distance of $0.1 \log$ MAR or better in both eyes. Exclusion criteria were uncontrolled glaucoma or retinal detachment, corneal disease, irregular corneal astigmatism, congenital bilateral cataract, macular degeneration or retinopathy, history of ocular inflammation, or other comorbidity that could affect surgical success (eg, high risk of intraoperative floppy iris syndrome), previous ocular trauma or patients requiring an IOL power outside the available range.

All patients provided written informed consent before enrollment. The study conformed to the tenets of the Declaration of Helsinki and the Regional Committee for Medical and Health Research Ethics (REK) approved the protocol.

\section{Intraocular lens}

The toric IOL Tecnis Symfony ZXT (Johnson \& Johnson Vision) is a one-piece extended range of vision lens, with an overall length of $13 \mathrm{~mm}$ and an optic diameter of $6 \mathrm{~mm}$ (Figure 1). The lens is made of foldable hydrophobic acrylic material and incorporates a frosted edge design with a $360^{\circ}$ posterior square edge. This IOL presents a biconvex, wavefront-designed anterior aspheric $(-0.27 \mu \mathrm{m})$ surface, whereas the posterior achromatic diffractive surface contains an echelette feature to extend the range of vision. It is available in spherical equivalent powers of 5.0-34.0 D in 0.5 diopter increments and in five cylindrical powers: 1.00 , $1.50,2.25,3.00$, and $3.75 \mathrm{D}$ (at the IOL plane).

\section{Preoperative assessment}

Before surgery, patients had a full ophthalmologic examination including measurement of monocular and binocular corrected distance visual acuity (CDVA) at $4 \mathrm{~m}$, using the Early Treatment Diabetic Retinopathy Study (ETDRS) charts under photopic conditions ( 85 candelas $[\mathrm{cd}] / \mathrm{m}^{2}$ ), autorefractometry (HRK 9000A; Huvitz Co., Ltd, Anyang, Korea) subjective refraction, slitlamp biomicroscopy, fundoscopy, keratometry, and corneal topography (Pentacam; Oculus Optikgeräte GmbH, Wetzlar, Germany).

Optical biometry (Lenstar 900; Haag Streit, Koeniz, Switzerland) was performed using the Barrett formula and the company's recommended A-constant of 119.3 for the toric 


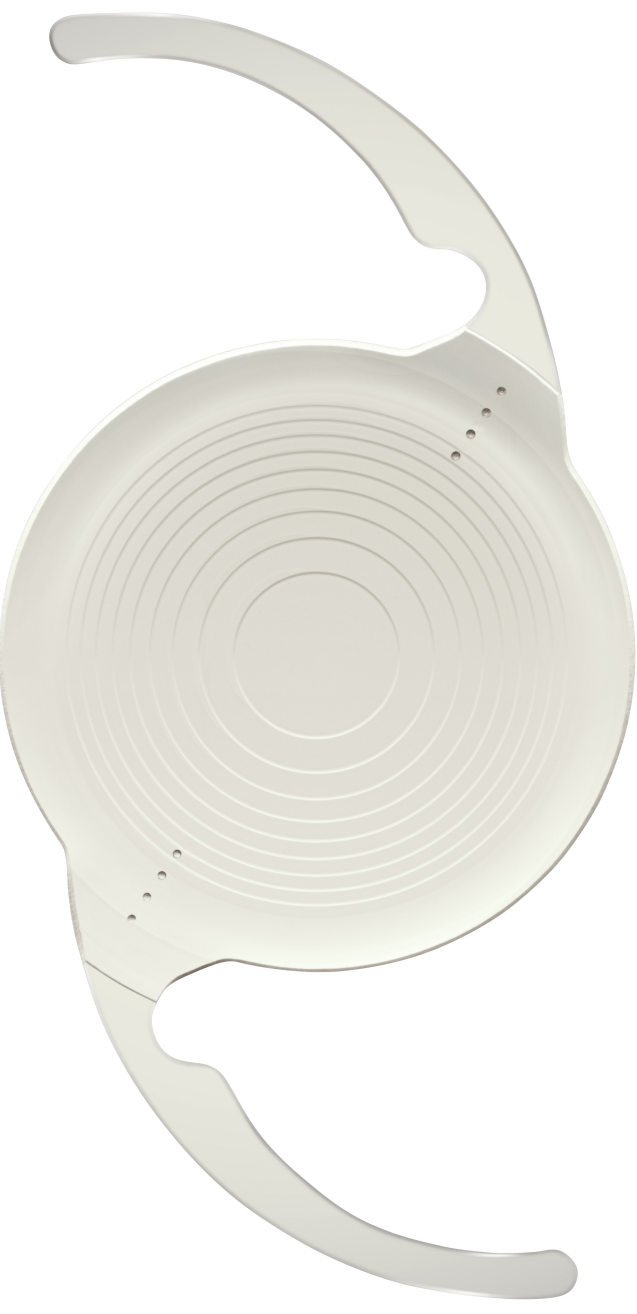

Figure I Tecnis Symfony ${ }^{\circledR}$ toric ZXT IOL.

IOL. Data for toric IOL calculation were taken directly from the Lenstar 900. The target postoperative spherical equivalent (SE) was aimed at emmetropia. Pentacam corneal topography map was used to confirm consistency of keratometry values and regularity of astigmatism. The IOL cylinder power and alignment axis were calculated using an online-calculator from the manufacturer (www.amoeasy.com/calc), taking into account the Lenstar 900 keratometry readings as well as data regarding to the position of the corneal incision. No surgically induced astigmatism was assumed because of the corneoscleral tunnel incision used.

\section{Surgical technique}

All surgeries were performed by the same experienced surgeon (KGG) under topical and intracameral (lidocain) anesthesia. Prior to surgery, reference photos were taken on all eyes using the Verion positioning system (Alcon Laboratories, Inc., Fort Worth, TX, USA) with the patients sitting in an upright position to avoid cyclotorsion errors.
A $2.2 \mathrm{~mm}$ self-sealing incision at the 12 o'clock position and a $5.5 \mathrm{~mm}$ continuous curvilinear capsulorhexis were created in all cases using the Verion video overlay as a guidance. The natural lens was removed by phacoemulsification. The toric lens was then implanted in the bag using the UNFOLDER Platinum 1 Series Screw-Style injector (Johnson \& Johnson Vision) and rotated clockwise to approximately 10 degrees short of the desired position. The desired axis of alignment was calculated using the Barrett formula and shown intraoperatively as a video overlay using the Verion guidance system. After IOL insertion and ophthalmic viscoelastic device (OVD) removal, the toric IOL was rotated with a second instrument to its final position. Postoperatively, patients were prescribed Tobrasone (Alcon Laboratories, Inc.) and Nevanac (Novartis Pharmaceuticals UK Ltd; Camberley, UK) eye drops four times daily tapered over 20 days.

\section{Postoperative assessment}

Patients were examined the day after surgery as well as 1 week and 3 months postoperatively. The postoperative examination protocol was identical to the preoperative one, with the additional evaluation of monocular and binocular uncorrected distance visual acuity (UDVA) at $4 \mathrm{~m}$, binocular uncorrected near visual acuity (UNVA) at $0.40 \mathrm{~m}$ and binocular uncorrected intermediate visual acuity (UIVA) at $0.66 \mathrm{~m}$. All visual acuity measurements were performed under photopic conditions $\left(85 \mathrm{~cd} / \mathrm{m}^{2}\right)$ using ETDRS charts designed for these distances. The alignment of the IOL was investigated and recorded using anterior segment optical coherence tomography (Casia SS-1000; Tomey Corp., Nagoya, Japan) at each follow-up visit.

At the last follow-up, low contrast visual acuity (LCVA) was measured using the HDC 9000PF Digital ETDRS Chart (Huvitz Co., Ltd, Korea). LCVA was tested monocularly and binocularly with the subject's best distance correction in place using a target at $4 \mathrm{~m}$ and a letter contrast of $10 \%$.

Patients were also asked to complete the 25-item version of the National Eye Institute Visual Function Questionnaire (NEI VFQ-25). ${ }^{22}$ This scientifically validated questionnaire consists of a base set of 25 questions representing 11 visionrelated constructs, plus a supplementary general health question rated by the patients on a scale from 0 (poor) to 100 (excellent). ${ }^{22}$ Additionally, patients were asked about their spectacle use after surgery: "How often do you need glasses to see comfortable at far, intermediate and near distances?" (0\%/25\%/50\%/75\%/100\% of time). Regarding patient satisfaction, patients were asked: "How satisfied are you with your spectacle free vision at far, intermediate and 
near distance"? (scale from 0: "not satisfied at all" to 10: "very satisfied"), "Would you choose the same lens again?" (yes/ no) and "Would you recommend this lens to your relatives and friends?" (yes/no). Patients were also asked about the perception of photic phenomena: "Are you bothered by any vision-related problems?" (yes/no) and "If you are bothered, please specify your problems" (glare/halos/starburst/others; each category graded as trace, mild, moderate and severe).

Likewise, the surgeon completed a short questionnaire, evaluating some surgical aspects for each case, including ease of IOL implantation and axis alignment, the overall satisfaction with the IOL and the achievement of target refraction, as well as the satisfaction with the visual outcome of the patient on a scale from 0 (not satisfied at all) to 10 (very satisfied).

\section{Statistical analysis}

All data were collected in an Excel database (Microsoft Office 2016; Microsoft Corporation, Redmond, WA, USA). Statistical analysis was performed using an Add-in for Microsoft Excel (WinSTAT, version 2012.1.0.96; R. Fitch Software, Bad Krozingen, Germany). Normality of all data samples was evaluated using the Shapiro-Wilk test. When parametric analysis was possible, the Student's $t$-test for paired data was performed for all comparisons between preoperative and postoperative examinations. Otherwise, when parametric analysis was not possible, the Wilcoxon rank sum test was applied to assess the significance of differences between examinations. Correlation coefficients (Pearson or Spearman, depending on whether normality condition could be assumed) were used to assess the correlation between different variables. A $P$-value of less than 0.05 was considered statistically significant.

\section{Results}

This study enrolled a total of 60 eyes from 30 patients with a mean age of $61 \pm 9.3$ years (range: 44-78). Patient demographics and implanted IOLs are summarized in Table 1. All patients completed the 3-month follow-up.

\section{Visual and refractive outcomes}

Table 2 shows the preoperative and postoperative visual and refractive data of the included patients. In the overall sample, a statistically significant improvement in monocular and binocular CDVA was found after surgery $(P<0.001)$. Changes in refractive sphere $(P=0.044)$, cylinder $(P<0.001)$ and spherical equivalent $(P=0.001)$ did also reach statistical significance 3 months postoperatively. Figure 2 displays the distribution of pre- and postoperative monocular distance
Table I Patient demographics and implanted IOLs

\begin{tabular}{ll}
\hline Parameters & Value \\
\hline Eyes $(\mathrm{n})$ & 60 \\
Patients (n) & \\
Total & 30 \\
Cataract surgery & 20 \\
Refractive lens exchange & 10 \\
Age (y) & \\
Mean (SD) & $61(9.3)$ \\
Range & $44-78$ \\
Male gender, $\mathrm{n}(\%)$ & $12(40 \%)$ \\
KI (mm) & \\
Mean (SD) & $7.93(0.31)$ \\
Range & $7.24-8.66$ \\
K2 (mm) & \\
Mean (SD) & $7.60(0.29)$ \\
Range & $6.96-8.34$ \\
Corneal astigmatism (D) & \\
Mean (SD) & $1.80(0.56)$ \\
Range & $0.88-3.29$ \\
Axial length (mm) & \\
Mean (SD) & $24.34(1.55)$ \\
Range & $20.68-27.65$ \\
IOL SE power (D) & \\
Mean (SD) & $19.38(4.44)$ \\
Range & $8.50-30.50$ \\
IOL model (n) & \\
ZXTI00 & 5 \\
ZXTI50 & 16 \\
ZXT225 & 24 \\
ZXT300 & 8 \\
ZXT375 & 7 \\
\hline Abreviatons: & \\
\hline
\end{tabular}

Abbreviations: $\mathrm{KI}$, corneal radii of curvature in the flattest meridian for the $3 \mathrm{~mm}$ central zone; K2, corneal radii of curvature in the steepest meridian for the $3 \mathrm{~mm}$ central zone; D, diopters; IOL, intraocular lens; SE, spherical equivalent.

visual acuities. Postoperatively, monocular UDVA was at least 0.3 logMAR (Snellen: 20/40) in 57 (98\%) eyes and at least $0.1 \log$ MAR (Snellen: 20/25) in 52 (90\%) eyes. In terms of postoperative binocular visual acuity, a total of $96 \%, 96 \%$, and $82 \%$ of patients achieved UDVA, UIVA, and UNVA of 0.1 logMAR (Snellen: 20/25) or better, respectively (Figure 3). Postoperative binocular CDVA was 0.1 logMAR (Snellen: 20/25) or better in all cases. Three months after surgery, mean monocular and binocular LCVA was 0.69 \pm 0.09 logMAR (range 0.44-0.94 logMAR) and 0.65 $0.09 \log$ MAR (range 0.44-0.86 logMAR), respectively.

Figure 4 shows the postoperative spherical equivalent. The SE of subjective refraction was within $\pm 0.50 \mathrm{D}$ of target correction in $43(74 \%)$ eyes, and within $\pm 1.00 \mathrm{D}$ in $56(97 \%)$ eyes.

Figure 5 displays the distribution of pre- and postoperative refractive astigmatism. After surgery, the refractive cylinder was within $0.50 \mathrm{D}$ of target correction in $51(88 \%)$ eyes and within $1.00 \mathrm{D}$ in $56(97 \%)$ eyes. 
Table 2 Preoperative and postoperative visual and refractive data ${ }^{\mathrm{a}}$

\begin{tabular}{|c|c|c|c|}
\hline Variable & Preoperative & 3 months postoperative & $P$-value \\
\hline \multirow[t]{2}{*}{ UDVA monocular (logMAR) } & - & $0.01(0.12)$ & - \\
\hline & & $0.00(-0.20$ to 0.40$)$ & \\
\hline \multirow[t]{2}{*}{ UDVA binocular (logMAR) } & - & $-0.05(0.11)$ & - \\
\hline & & $-0.10(-0.20$ to 0.30$)$ & \\
\hline \multirow[t]{2}{*}{ Sphere (D) } & $-0.91(3.20)$ & $0.06(0.44)$ & 0.044 \\
\hline & $-0.88(-10.00$ to 5.25$)$ & $0.00(-1.75$ to 1.00$)$ & \\
\hline \multirow[t]{2}{*}{ Cylinder (D) } & $-1.72(1.01)$ & $-0.33(0.37)$ & $<0.001$ \\
\hline & $-1.50(-4.00$ to 0.00$)$ & $-0.25(-1.75$ to 0.00$)$ & \\
\hline \multirow[t]{2}{*}{ Spherical equivalent (D) } & $-1.77(3.26)$ & $-0.1 I(0.5 \mathrm{I})$ & 0.001 \\
\hline & $-2.00(-10.75$ to 4.75$)$ & $0.00(-2.38$ to 0.75$)$ & \\
\hline \multirow[t]{2}{*}{ CDVA monocular (logMAR) } & $0.10(0.14)$ & $-0.04(0.07)$ & $<0.001$ \\
\hline & $0.10(-0.10$ to 0.40$)$ & $0.00(-0.20$ to 0.20$)$ & \\
\hline \multirow[t]{2}{*}{ CDVA binocular (logMAR) } & $0.04(0.13)$ & $-0.08(0.07)$ & $<0.001$ \\
\hline & $0.00(-0.20$ to 0.30$)$ & $-0.10(-0.20$ to 0.10$)$ & \\
\hline \multirow[t]{2}{*}{ UIVA binocular (logMAR) } & - & $-0.03(0.08)$ & - \\
\hline & & $0.00(-0.10$ to 0.20$)$ & \\
\hline \multirow[t]{2}{*}{ UNVA binocular (logMAR) } & - & $0.09(0.10)$ & - \\
\hline & & $0.10(-0.10$ to 0.40$)$ & \\
\hline
\end{tabular}

Note: ${ }^{2}$ Values reported as mean (SD), median (range).

Abbreviations: D, diopters; UDVA, uncorrected distance visual acuity; CDVA, corrected distance visual acuity; UIVA, uncorrected intermediate visual acuity; UNVA, uncorrected near visual acuity.

As verified by slitlamp examination 3 months after surgery, there was no significant posterior capsule opacification, IOL tilt or decentration influencing the visual or refractive outcomes.

\section{Rotational stability}

Data on IOL rotation and IOL misalignment were available in $52(87 \%)$ and 51 eyes (85\%), respectively. Analysis of the toric IOL axis position showed a median rotation of 2.0 degrees (range $0.0-16.0$ degrees) from the end of surgery to the last follow-up. IOL rotation was within 5 degrees in $87 \%(n=45)$, and within 10 degrees in $96 \%(n=50)$ of eyes. Three months postoperative, median IOL axis misalignment from the preoperative intended axis was 3.0 degrees (range $0.0-11.0$ degrees). The alignment was within 5 degrees of the intended axis in $88 \%(n=45)$ of eyes and within 10 degrees

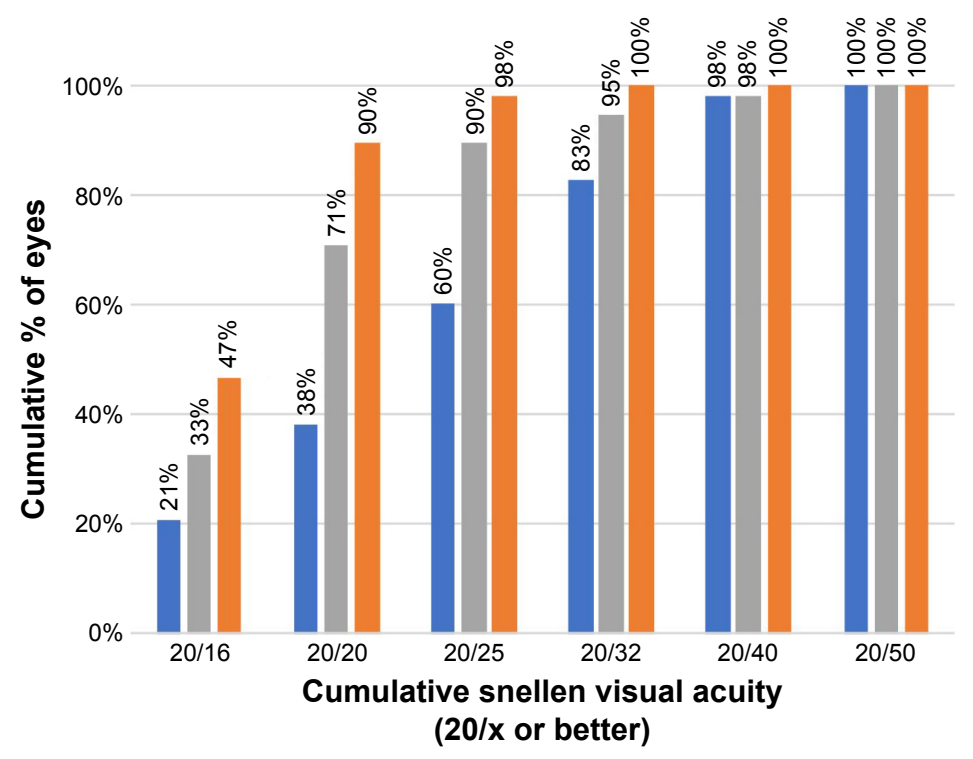

$\square$ Preop CDVA $\quad$ Postop UDVA $\quad$ Postop CDVA

Figure 2 Distribution of pre- and postoperative monocular visual outcomes.

Abbreviations: CDVA, corrected distance visual acuity; UDVA, uncorrected distance visual acuity. 


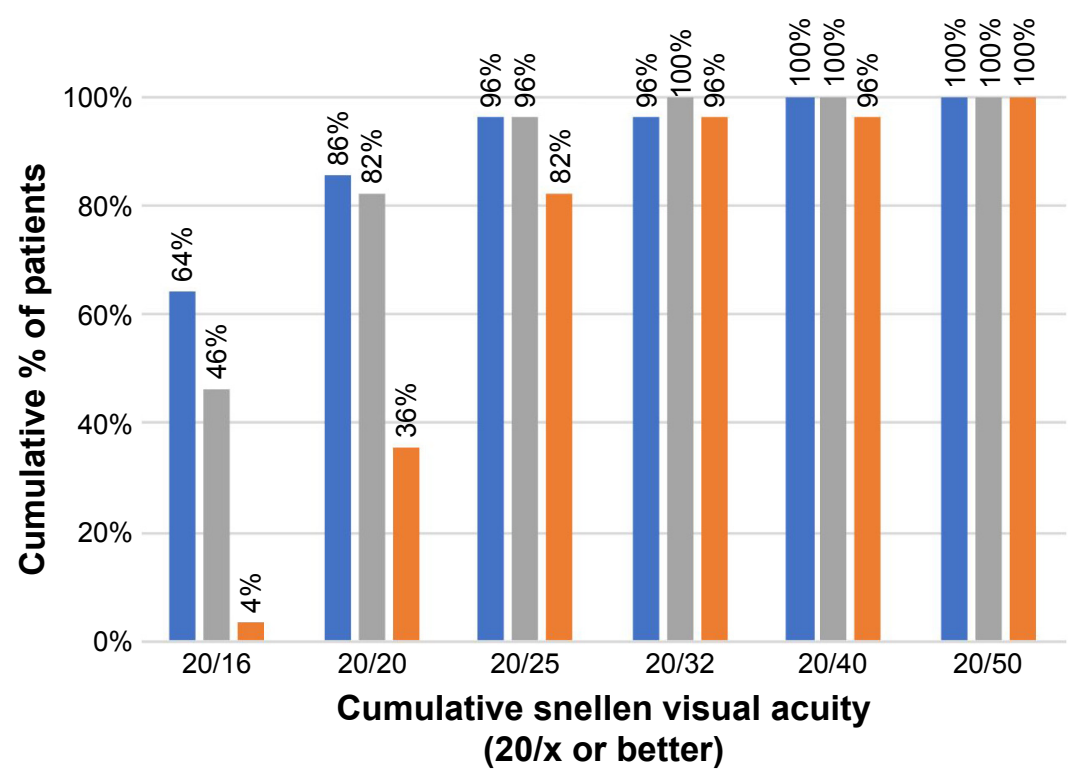

UDVA UIVA UNVA

Figure 3 Distribution of 3-month postoperative binocular visual outcomes.

Abbreviations: UDVA, uncorrected distance visual acuity; UIVA, uncorrected intermediate visual acuity; UNVA, uncorrected near visual acuity.

in $98 \%(n=50)$ of eyes. There was no correlation between the postoperative IOL rotation and the specific axial length of the eyes $(r=-0.06 ; P=0.346)$.

\section{Quality of life}

The postoperative NEI VFQ-25 scores are presented in Table 3. Results show a mean overall quality-of-life assessment of $94.1 \pm 5.4$ (range 50-100) points. Vision during distance and near activities was rated by the patients with a mean score of $97.8 \pm 4.3$ and $91.7 \pm 10.8$, respectively. Within all vision-related subscales, the section of driving abilities yielded the lowest valuation but still with a satisfactory mean score of $87.5 \pm 14.1$.

\section{Photic phenomena}

After 3 months, most patients $(89 \%, \mathrm{n}=25)$ did not perceive or were not bothered by any photic phenomena. One patient (4\%) reported slight difficulty in night driving whereas a

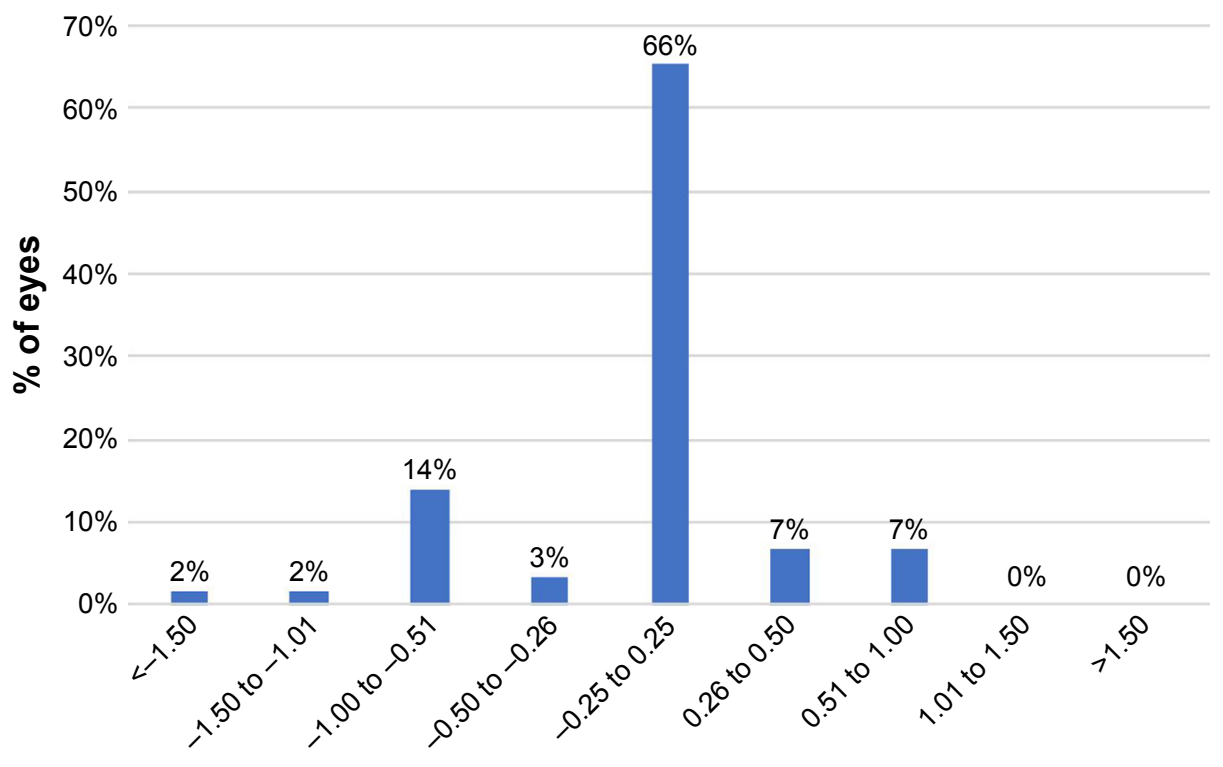

Postoperative spherical equivalent (D)

Figure 4 Distribution of 3-month postoperative spherical equivalent.

Abbreviation: $\mathrm{D}$, diopters. 


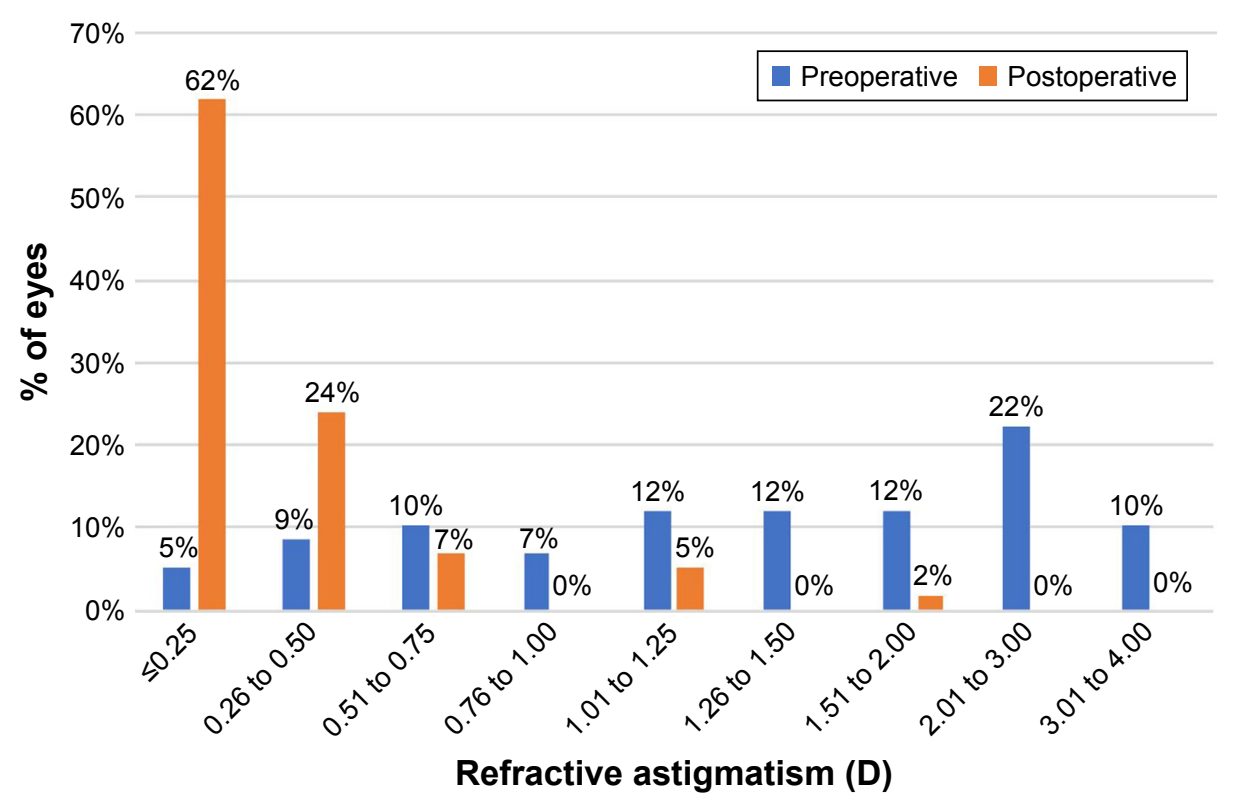

Figure 5 Pre- and postoperative refractive astigmatism.

Abbreviation: $\mathrm{D}$, diopters.

second patient (4\%) stated mild symptoms of halos and traces of glare. Postoperative halos graded as "moderate" were reported by only one patient (4\%).

\section{Patient satisfaction and spectacle use}

The postoperative median patient satisfaction scores for distance, intermediate, and near vision were 9.0 (range 5-10), 9.0 (range 6-10), and 8.0 (range 2-9), respectively.

Patients were also asked to report the frequency of spectacle use for different distances (Figure 6). Overall, half of the patients $(n=14)$ stated never wearing glasses for any distance. Furthermore, $86 \%(\mathrm{n}=24), 96 \%(\mathrm{n}=27)$, and $64 \%$ $(n=18)$ of patients were totally spectacle independent for distance, intermediate, and near visual tasks, respectively. Nearly all patients $(96 \% ; n=27)$, except the one complaining

Table 3 Postoperative NEI VFQ-25 scores

\begin{tabular}{lll}
\hline NEI VFQ-25 subscale & Mean (SD) & Median (range) \\
\hline General health & $77.5(13.5)$ & $75(50-100)$ \\
General vision & $85.3(13.6)$ & $80(60-100)$ \\
Ocular pain & $97.5(6.0)$ & $100(75-100)$ \\
Near activities & $91.7(10.8)$ & $96(58-100)$ \\
Distance activities & $97.8(4.3)$ & $100(83-100)$ \\
Social functioning (vision specific) & $100(0.0)$ & $100(100)$ \\
Mental health (vision specific) & $95.6(5.4)$ & $100(81-100)$ \\
Role difficulties (vision specific) & $96.7(6.4)$ & $100(75-100)$ \\
Dependency (vision specific) & $100(0.0)$ & $100(100)$ \\
Driving & $87.5(14.1)$ & $88(50-100)$ \\
Color vision & $100(0.0)$ & $100(100)$ \\
Peripheral vision & $100(0.0)$ & $100(100)$ \\
NEI VFQ-25 (overall) & $\mathbf{9 4 . 1 ( 5 . 4 )}$ & $100(50-100)$ \\
\hline
\end{tabular}

Abbreviation: NEI VFQ-25, 25-item version of the National Eye Institute Visual Function Questionnaire. of moderate halos, would choose the same lens model again and would also recommend this IOL to friends or family members.

\section{Surgeon assessment}

Surgeon satisfaction scores were excellent, with a median value of 10.0, for the ease of lens implantation (range 9-10), the ease of axis alignment (range 9-10), the achievement of target refraction (range 6-10), and the overall satisfaction with the IOL (range 8-10). The median score for surgeon satisfaction with the visual outcome of the patient was 9.0, ranging from 7 to 10 points.

\section{Complications}

After the 3-month follow-up, two eyes required surgical re-alignment of the IOL axis due to severe postoperative rotation of 36 and 28 degrees, both resulting in a refractive cylinder of 2.25 D and therefore poor uncorrected visual acuity. After realignment, the two IOL axes remained stable in the desired position and UDVA improved to $-0.02 \log$ MAR in both eyes. One of these two eyes needed an additional limbal relaxation incision to reduce residual corneal astigmatism. One eye developed cystoid macular edema (CME) after surgery with full recovery confirmed at the last follow-up. No further complications were found during the follow-up period.

\section{Discussion}

To our knowledge, this is the first study reporting the clinical outcomes with the toric version of the ERV IOL Tecnis Symfony. In our prospective study, the Symfony toric IOL 


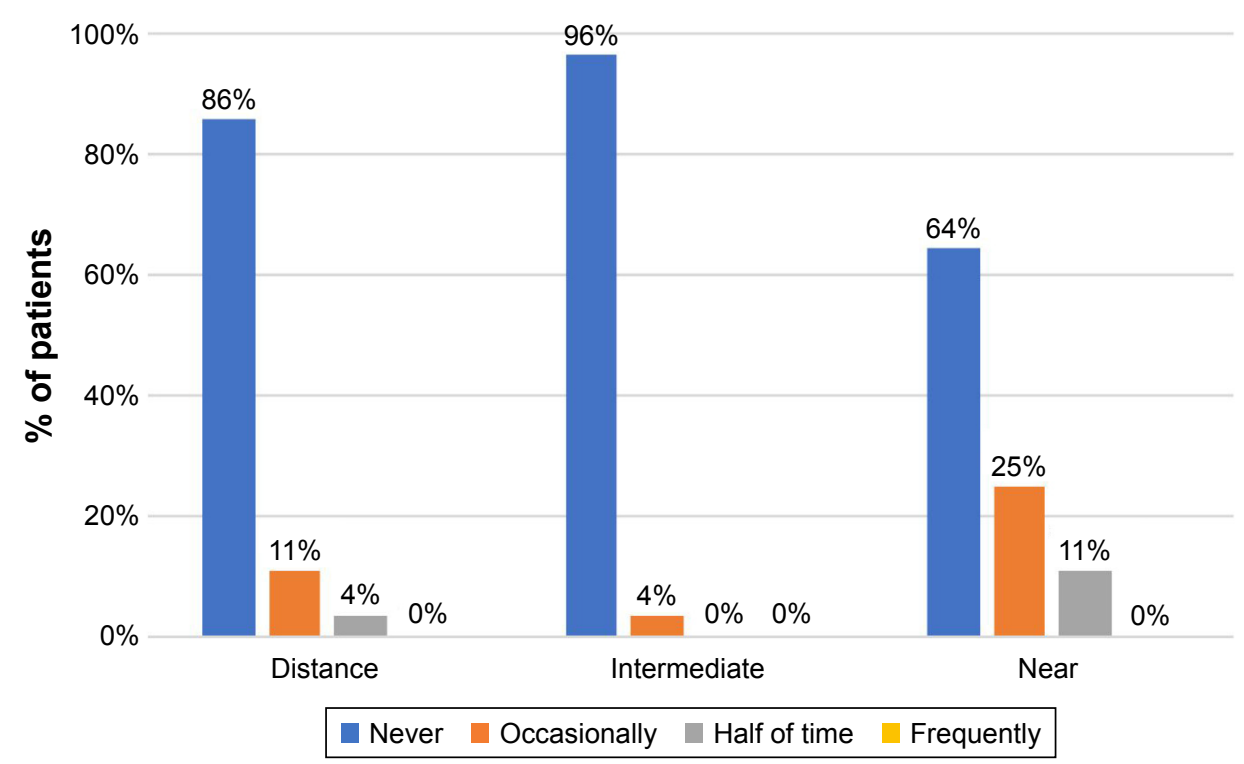

Figure 6 Frequency of spectacle use 3 months after surgery.

provided successful visual restoration after cataract surgery or RLE with excellent visual outcomes for far and intermediate distances in combination with a functional near vision. At the 3-month follow-up, mean binocular UDVA, UIVA, and UNVA (logMAR) were $-0.05 \pm 0.11,-0.03 \pm 0.08$, and $0.09 \pm 0.10$, respectively. Our outcomes for UDVA are consistent with those reported for the spherical Symfony IOL model reported by several authors. ${ }^{11-18}$ However, most of these aforementioned studies showed a lower binocular UNVA, ranging from $0.19^{11,12}$ to $0.11 \log$ MAR, ${ }^{17}$ and also a lower UIVA compared to our findings. ${ }^{13,16-18}$ Regarding UNVA, only Monaco et al ${ }^{18}$ reported comparable binocular outcomes $(0.07 \pm 0.08)$ in a series of 40 eyes 4 months postoperatively. We found similar ${ }^{14}$ or even better outcomes for UIVA in our series as compared to published data of the non-toric Symfony IOL. ${ }^{13,16-18}$ Monaco et $\mathrm{al}^{18}$ reported a mean UIVA of $0.27 \pm 0.08 \operatorname{logMAR}$, and also Ruiz-Mesa et al ${ }^{13}$ $(0.09 \pm 0.08)$, Cochener et $\mathrm{al}^{16}(0.12 \pm 0.16)$, and de Medeiros et $\mathrm{al}^{17}(0.20 \pm 0.05)$ found UIVA outcomes lower than ours. The described differences in UIVA and UNVA among these studies and also compared to our study may be attributed to factors such as differences in visual acuity measurement methods, residual refractive errors and study populations. Uncorrected visual acuity was higher for intermediate and lower for near vision when comparing our results to studies with trifocal lenses. ${ }^{13,17,18}$ A recently published study ${ }^{17}$ found a mean binocular UIVA of $0.14 \pm 0.05 \log$ MAR after implantation of a trifocal IOL (AcrySof IQ PanOptix; Alcon Laboratories, Inc.); mean binocular UNVA was $-0.03 \pm 0.04$ $\log$ MAR. The mean binocular CDVA was $-0.08 \pm 0.07$ $\log$ MAR in our study, which is comparable to previous reports about the spherical Tecnis Symfony IOL and modern trifocal lenses. ${ }^{11,12,16-18}$

Residual refractive astigmatism appears to be a crucial factor for the retreatment rate after multifocal IOL implantation. ${ }^{23}$ However, in a study comparing the impact of simulated residual astigmatism with different types of bifocal, trifocal, and the Symfony ERV IOL, the author reported that residual cylinders after implantation of the Tecnis Symfony IOL up to $1.0 \mathrm{D}$ have only a minimal, clinically not relevant impact on visual acuity outcomes or patient satisfaction. ${ }^{24}$ In comparison to diffractive bifocal and trifocal IOLs, the ERV IOL showed a better tolerance to postoperative residual cylinder errors. ${ }^{24}$ In our study, the residual refractive cylinder was within $0.50 \mathrm{D}$ of target refraction in $88 \%$ of eyes and within $1.00 \mathrm{D}$ in $97 \%$ of eyes 3 months after surgery. These results were comparable to those reported with different types of multifocal toric IOLs. ${ }^{25-27}$

In our series, the postoperative rotational stability of the IOL was high, with an absolute median toric IOL axis rotation of only 2.00 degrees from surgery to month 3 ; the median IOL misalignment from the preoperative calculated target axis was 3.00 degrees. Marques et $\mathrm{a}^{26}$ evaluated the multifocal toric IOL Tecnis ZMT (Johnson \& Johnson Vision) and found a mean toric IOL axis rotation of $3.12 \pm 3.16$ degrees (range: $0-8$ degrees) at month 1 and $3.18^{\circ} \pm 3.28^{\circ}$ (range: $0-8$ degrees) at month 6. Mojzis et $\mathrm{al}^{27}$ reported the 3-months outcomes after implantation of the trifocal toric IOL AT Lisa tri (Carl Zeiss Meditec, Jena, Germany) in 30 eyes and IOL rotation was 0 degrees in $40 \%$, between 1 and 3 degrees in $53 \%$, and 
between 4 and 5 degrees in 7\%. In our study, only two eyes required a second surgery to re-align the axis due to significant IOL rotation (36 and 28 degrees) 3 months after surgery. As reported in previous studies, long axial length is one risk factor associated with postoperative toric IOL rotation. ${ }^{28,29}$ Both affected eyes of the present study showed axial lengths greater than $25 \mathrm{~mm}$ (26.44 $\mathrm{mm}$ and $25.50 \mathrm{~mm}$ ) preoperatively which may be considered as a possible cause for lens rotation, although we found no correlation of axial length and IOL rotation in the study population ( $r=-0.06 ; P=0.346)$.

The good level of refractive predictability as well as the high rotational stability of the lens observed in our series were one of the main factors leading to a high rate of postoperative spectacle independence. Overall, 86\%, 96\% and 64\% of patients were totally spectacle independent for distance, intermediate and near visual tasks, respectively. Regarding near vision, $25 \%$ of patients stated that they need reading glasses only occasionally and $11 \%$ of patients chose the option "half of time". These results were comparable to those reported with the spherical version of the Tecnis Symfony ERV lens ${ }^{16,18}$ but inferior compared to the outcomes with modern trifocal lenses. ${ }^{13,18,26}$ Monaco et $\mathrm{al}^{18}$ assessed the outcomes for the non-toric Tecnis Symfony IOL and the trifocal AcrySof IQ PanOptix lens in 20 patients per group. In the trifocal group, $90 \%$ of patients were completely spectacle independent for near tasks in contrast to $75 \%$ in the ERV group, but differences between groups did not reach statistical significance. $^{18}$

In contrast to ERV or EDOF IOLs, which are designed to lengthen the focus of the eye resulting in one elongated focal point, ${ }^{16}$ multifocal lenses use the principle of simultaneous vision in which the light is split into two or more focal points. As a result, multifocal IOLs offer the prerequisite for better near visual acuity compared to ERV lenses but simultaneously increase the risk of disturbing postoperative photic phenomena. In our series, most patients (89\%) did not perceive or were not bothered by any photic phenomena. One patient (4\%) reported slight difficulties in night driving, a second patient stated mild symptoms of halos and traces of glare, and a third patient reported moderate halos. These results are superior to studies evaluating modern multifocal toric lenses. ${ }^{25-27}$

Only a few studies addressed quality of life after multifocal IOL implantation using validated instruments such as NEI VFQ-25. Mojzis et $\mathrm{al}^{27}$ used the NEI VFQ-14 questionnaire to assess quality of life after implantation of AT Lisa tri toric IOLs and found a mean value of 92.5. Our results also showed an excellent overall quality-of-life valuation of
94.1 (mean), and all vision-related subscales reached median values $\geq 80$, demonstrating a high overall patient satisfaction. Nearly all patients (96\%) stated that they would choose the same IOL model again. As suggested by the results in the Concerto Study, ${ }^{16}$ patient satisfaction after implantation of Symfony ERV IOLs may be further improved by the adoption of an intended micro-monovision technique to maximize UNVA outcomes. Additionally, surgeon satisfaction scores for intraoperative IOL handling and postoperative patient outcomes were high in our series. This is in accordance with results reported for the non-toric Symfony IOL in a large study including 411 patients. $^{16}$

\section{Conclusion}

Implantation of the Tecnis Symfony toric ERV IOL provided an effective restoration of distance and intermediate vision together with an efficient near vision and high spectacle independence after cataract/RLE in eyes with small to moderate levels of corneal astigmatism. This visual restoration is accompanied by high levels of patient satisfaction and a minimal incidence of photic phenomena. Future studies should address additional parameters, such as defocus curves and contrast sensitivity.

\section{Disclosure}

The author reports no conflicts of interest in this work.

\section{References}

1. Rosen E, Alió JL, Dick HB, Dell S, Slade S. Efficacy and safety of multifocal intraocular lenses following cataract and refractive lens exchange: Metaanalysis of peer-reviewed publications. J Cataract Refract Surg. 2016;42(2):310-328.

2. de Silva SR, Evans JR, Kirthi V, Ziaei M, Leyland M. Multifocal versus monofocal intraocular lenses after cataract extraction. Cochrane Database Syst Rev. 2016;12:CD003169.

3. Shah S, Peris-Martinez C, Reinhard T, Vinciguerra P. Visual outcomes after cataract surgery: multifocal versus monofocal intraocular lenses. $J$ Refract Surg. 2015;31(10):658-666.

4. Gundersen KG, Potvin R. Comparison of visual outcomes after implantation of diffractive trifocal toric intraocular lens and a diffractive apodized bifocal toric intraocular lens. Clin Ophthalmol. 2016;10:455-461.

5. Alfonso JF, Fernández-Vega L, Amhaz H, Montés-Micó R, Valcárcel B, Ferrer-Blasco T. Visual function after implantation of an aspheric bifocal intraocular lens. J Cataract Refract Surg. 2009;35(5):885-892.

6. Jafarinasab MR, Feizi S, Baghi AR, Ziaie H, Yaseri M. Aspheric versus spherical posterior chamber intraocular lenses. J Ophthalmic Vis Res. 2010;5(4):217-222.

7. Zhao H, Mainster MA. The effect of chromatic dispersion on pseudophakic optical performance. Br J Ophthalmol. 2007;91(9):1225-1229.

8. Negishi K, Ohnuma K, Hirayama N, Noda T; Policy-Based Medical Services Network Study Group for Intraocular Lens and Refractive Surgery. Effect of chromatic aberration on contrast sensitivity in pseudophakic eyes. Arch Ophthalmol. 2001;119(8):1154-1158.

9. Weeber HA, Meijer ST, Piers PA. Extending the range of vision using diffractive intraocular lens technology. J Cataract Refract Surg. 2015;41(12):2746-2754. 
10. Weeber HA, Piers PA. Theoretical performance of intraocular lenses correcting both spherical and chromatic aberration. J Refract Surg. 2012;28(1):48-52.

11. Kaymak H, Höhn F, Breyer DR, et al. Functional results 3 months after implantation of an "extended range of vision" intraocular lens. Klin Monbl Augenheilkd. 2016;233(8):923-927.

12. Ruiz-Mesa R, Abengózar-Vela A, Ruiz-Santos M. A comparative study of the visual outcomes between a new trifocal and an extended depth of focus intraocular lens. Eur J Ophthalmol. 2018;28(2):182-187.

13. Ruiz-Mesa R, Abengózar-Vela A, Aramburu A, Ruiz-Santos M. Comparison of visual outcomes after bilateral implantation of extended range of vision and trifocal intraocular lenses. Eur J Ophthalmol. 2017;27(4):460-465.

14. Attia MSA, Auffarth GU, Kretz FTA, et al. Clinical evaluation of an extended depth of focus intraocular lens with the Salzburg reading desk. J Refract Surg. 2017;33(10):664-669.

15. Pedrotti E, Bruni E, Bonacci E, Badalamenti R, Mastropasqua R, Marchini G. Comparative analysis of the clinical outcomes with a monofocal and an extended range of vision intraocular lens. J Refract Surg. 2016;32(7):436-442.

16. Cochener B; Concerto Study Group, Group CS. Clinical outcomes of a new extended range of vision intraocular lens: International Multicenter Concerto Study. J Cataract Refract Surg. 2016;42(9):1268-1275.

17. de Medeiros AL, de Araújo Rolim AG, Motta AFP, et al. Comparison of visual outcomes after bilateral implantation of a diffractive trifocal intraocular lens and blended implantation of an extended depth of focus intraocular lens with a diffractive bifocal intraocular lens. Clin Ophthalmol. 2017;11:1911-1916.

18. Monaco G, Gari M, di Censo F, Poscia A, Ruggi G, Scialdone A. Visual performance after bilateral implantation of 2 new presbyopia-correcting intraocular lenses: Trifocal versus extended range of vision. J Cataract Refract Surg. 2017;43(6):737-747.

19. Black S. Successful restoration of visual acuity with an extended range of vision intraocular lens after multifocal laser ablation. Case Rep Ophthalmol. 2016;7(3):193-197.
20. Hayashi K, Manabe S, Yoshida M, Hayashi H. Effect of astigmatism on visual acuity in eyes with a diffractive multifocal intraocular lens. J Cataract Refract Surg. 2010;36(8):1323-1329.

21. Hoffmann PC, Hütz WW. Analysis of biometry and prevalence data for corneal astigmatism in 23,239 eyes. J Cataract Refract Surg. 2010; 36(9):1479-1485.

22. Mangione CM, Lee PP, Gutierrez PR, et al. Development of the 25-item National Eye Institute Visual Function Questionnaire. Arch Ophthalmol. 2001;119(7):1050-1058.

23. Gundersen KG, Makari S, Ostenstad S, Potvin R. Retreatments after multifocal intraocular lens implantation: an analysis. Clin Ophthalmol. 2016;10:365-371

24. Carones F. Residual astigmatism threshold and patient satisfaction with bifocal, trifocal and extended range of vision intraocular lenses (IOLs). Open J Ophthalmol. 2017;07(01):1-7.

25. Kretz FT, Breyer D, Klabe K, et al. Clinical outcomes after implantation of a trifocal toric intraocular lens. J Refract Surg. 2015;31(8): 504-510.

26. Marques EF, Ferreira TB, Simões P. Visual performance and rotational stability of a multifocal toric intraocular lens. J Refract Surg. 2016;32(7): 444-450.

27. Mojzis P, Majerova K, Plaza-Puche AB, Hrckova L, Alio JL. Visual outcomes of a new toric trifocal diffractive intraocular lens. J Cataract Refract Surg. 2015;41(12):2695-2706.

28. Shah GD, Praveen MR, Vasavada AR, Vasavada VA, Rampal G, Shastry LR. Rotational stability of a toric intraocular lens: influence of axial length and alignment in the capsular bag. J Cataract Refract Surg. 2012;38(1):54-59.

29. Zhu X, He W, Zhang K, Lu Y. Factors influencing 1-year rotational stability of AcrySof Toric intraocular lenses. Br J Ophthalmol. 2016; 100(2):263-268.
Clinical Ophthalmology

\section{Publish your work in this journal}

Clinical Ophthalmology is an international, peer-reviewed journal covering all subspecialties within ophthalmology. Key topics include: Optometry; Visual science; Pharmacology and drug therapy in eye diseases; Basic Sciences; Primary and Secondary eye care; Patient Safety and Quality of Care Improvements. This journal is indexed on Submit your manuscript here: http://www.dovepress.com/clinical-ophthalmology-journal

\section{Dovepress}

PubMed Central and CAS, and is the official journal of The Society of Clinical Ophthalmology (SCO). The manuscript management system is completely online and includes a very quick and fair peer-review system, which is all easy to use. Visit http://www.dovepress.com/ testimonials.php to read real quotes from published authors. 